Published in final edited form as:

ACS Chem Biol. 2016 September 16; 11(9): 2499-2505. doi:10.1021/acschembio.6b00182.

\title{
EPI-001, a compound active against castration-resistant prostate cancer, targets transactivation unit 5 of the androgen receptor
}

\author{
Eva De Mol ${ }^{1}$, R. Bryn Fenwick ${ }^{1}$, Christopher T. W. Phang ${ }^{1}$, Victor Buzón ${ }^{2}$, Elzbieta Szulc ${ }^{1}$, \\ Alex de la Fuente ${ }^{1}$, Albert Escobedo ${ }^{1}$, Jesús García ${ }^{1}$, Carlos W. Bertoncini ${ }^{1}$, Eva \\ Estébanez-Perpiñá2 ${ }^{2}$ lain J. McEwan ${ }^{3}$, Antoni Riera ${ }^{1,4}$, and Xavier Salvatella ${ }^{1,5,{ }^{*}}$ \\ ${ }^{1}$ Institute for Research in Biomedicine (IRB Barcelona), The Barcelona Institute of Science and \\ Technology, Baldiri Reixac 10, 08028 Barcelona, Spain \\ 2Departament de Bioquímica i Biología Molecular, Universitat de Barcelona and Institute of \\ Biomedicine of the University of Barcelona (IBUB), Baldiri Reixac 10, 08028 Barcelona, Spain \\ ${ }^{3}$ Institute of Medical Sciences, School of Medicine, Medical Sciences and Nutrition, University of \\ Aberdeen, IMS Building, Foresterhill, Aberdeen AB25 2ZD, Scotland, United Kingdom \\ ${ }^{4}$ Departament de Química Orgánica, Universitat de Barcelona, Martí i Franqués 1-11, 08028 \\ Barcelona, Spain \\ ${ }^{5}$ ICREA, Passeig Lluís Companys 23, 08010 Barcelona, Spain
}

\begin{abstract}
Castration-resistant prostate cancer is the lethal condition suffered by prostate cancer patients that become refractory to androgen deprivation therapy. EPI-001 is a recently identified compound active against this condition that modulates the activity of the androgen receptor, a nuclear receptor that is essential for disease progression. The mechanism by which this compound exerts its inhibitory activity is however not yet fully understood. Here we show, by using high resolution solution nuclear magnetic resonance spectroscopy, that EPI-001 selectively interacts with a partially folded region of the transactivation domain of the androgen receptor, known as transactivation unit 5, that is key for the ability of prostate cells to proliferate in the absence of androgens, a distinctive feature of castration-resistant prostate cancer. Our results can contribute to the development of more potent and less toxic novel androgen receptor antagonists for treating this disease.
\end{abstract}

\section{Introduction}

Prostate cancer (PC) is the second most common cancer in men and can be cured by surgery or radiotherapy in ca 70\% of cases. The first line of pharmacological treatment for the remaining cases targets the androgen receptor (AR) because prostate cells depend on its activation by androgens for their growth and proliferation 1 . Activation can be prevented by combining androgen deprivation therapy, which inhibits the secretion of androgens by the

* to whom correspondence should be addressed: +34 934020459, xavier.salvatella@ irbbarcelona.org. 
testes, with the administration of antagonists that competitively bind to the binding site of androgens in the ligand-binding domain (LBD) of AR2.

Two to three years into this treatment PC patients inevitably develop castration-resistant prostate cancer (CRPC) as prostate cancer cells acquire the ability to activate AR at low levels of circulating androgens and in the presence of antagonists 3 . The mechanisms of aberrant activation are not well understood but appear to include the amplification of the $A R$ gene and AR overexpression, the expression of constitutively active AR splice variants lacking the LBD, cell signaling cross-talk and mutations in both AR and transcriptional coregulators 4 .

AR is a large multi-domain protein composed of globular ligand- and DNA-binding domains (LBD and DBD) and an N-terminal transactivation domain (NTD) that is intrinsically disordered (ID)5,6 (Fig. 1a). The function of the NTD (residues 1 to 558) is to recruit the basal transcription machinery by binding to general transcription factors either directly or assisted by transcriptional co-activators1. These protein-protein interactions are thought to cause the folding of binding motifs in a region of the NTD called activation function 1 (AF-1) that has not yet been characterized at high resolution (Fig. 1a,b)6. Inhibiting these interactions is considered a potential therapeutic approach for both PC and CRPC7, but the NTD has not been considered a suitable target for drug discovery due to its apparent lack of persistent secondary and tertiary structure.

The development of drugs that interact with ID regions has however recently been met with some success8,9, and has shown that targeting them with small molecules may be a viable therapeutic approach10,11. A particularly important development in this area was the recent discovery of EPI-001, an experimental drug for the treatment of CRPC identified by phenotypic screening that is efficacious both in cell lines and in an animal model of this disease12. In vivo EPI-001 binds irreversibly to the AR NTD and weakens its interaction with general transcription factors and transcriptional co-activators 13.

The discovery of EPI-001 represents an important milestone and a derivative of this compound is currently in clinical trials for CRPC (NCT02606123). The lack of a detailed understanding of the structural features of the domain and of the mechanism of action of this class of compounds represents, however, a hurdle for the rational design of optimized inhibitors. Here we reveal, by using solution nuclear magnetic resonance (NMR) spectroscopy, the structural properties of the ID regions of the NTD predicted to have a high propensity to fold and show that EPI-001 targets a region of sequence, known as transcription activation unit 5 (Tau-5), that is key for the ability of prostate cells to proliferate in the absence of androgens.

\section{Methods}

\section{Protein expression and purification}

The DNA sequences coding for human WT AR residues 265 to 340 (AF-1*265-340), 330 to 448 (Tau-5*) and 142 to 448 (AF-1*) were cloned into Gateway pDEST17 vectors (Invitrogen) with an $\mathrm{N}$-terminal $\mathrm{His}_{6}$-tag and a TEV cleavage site. Transformed E. coli 
Rosetta cells were grown at $37^{\circ} \mathrm{C}$ in $\mathrm{LB}$ medium for the production of non-isotopically labeled protein. For single $\left({ }^{15} \mathrm{~N}\right)$ or double $\left({ }^{15} \mathrm{~N},{ }^{13} \mathrm{C}\right)$ isotopic labeling, cells were grown in minimal MOPS medium14 containing ${ }^{15} \mathrm{NH}_{4} \mathrm{Cl}$ or ${ }^{15} \mathrm{NH}_{4} \mathrm{Cl}$ and ${ }^{13} \mathrm{C}$-glucose, respectively. The AF-1* fusion protein accumulated in inclusion bodies which were solubilized in lysis buffer containing $8 \mathrm{M}$ urea and fragmented by a pass through a cell disruptor at $25 \mathrm{kpsi}$. The fusion protein was purified by $\mathrm{Ni}^{2+}$ affinity chromatography in urea, which was removed by two dialysis steps, after which the His $_{6}$-tag was cleaved by the TEV protease. The cleaved $\mathrm{AF}-1 *$ was further purified by reverse $\mathrm{Ni}^{2+}$ affinity and size exclusion chromatography in 20 $\mathrm{mM}$ sodium phosphate buffer with $1 \mathrm{mM}$ tris(2-carboxyethyl)phosphine hydrochloride (TCEP) at $\mathrm{pH}$ 7.4.

\section{Peptides}

The synthesis of peptides R1, R2 and R3 (See SI) was performed by solid phase peptide synthesis by GenScript (peptide R1) or by ICTS NANBIOSIS, more specifically by the peptide synthesis unit of the CIBER in bioengineering, biomaterials \& nanomedicine (CIBER-BBN) at the Barcelona science park (peptides R2 and R3). The lyophilized peptides were dissolved in deionized water and the $\mathrm{pH}$ of the resulting solution was adjusted by addition of a concentrated $\mathrm{NaOH}$ solution. The concentration of these solutions was determined by amino acid analysis. The absence of intermolecular disulfide bonds in the R2 peptide, which contains one Cys residue, was confirmed by mass spectrometry (MS).

\section{Chemical synthesis of EPI-001 and stereoisomers}

EPI-001 contains two stereogenic centers and can therefore be found as two pairs of enantiomers. We synthesized the four isomers with high diastereo- and enantioselectivity (Chiral HPLC). The synthesis followed the sequence detailed in Fig. S1 of the SI. Bisphenol A was treated with enantiomerically pure glycidol. The resulting diol was protected as dimethyl acetal and the free phenol was allowed to react with another isomer of glycidol. The corresponding diol was transformed into the epoxide and opened with $\mathrm{CeCl}_{3}$. The treatment gave the final product by concomitant deprotection of the acetal. Full experimental details as well as the complete characterization of all isomers can be found in the SI.

\section{NMR}

The assignment of AF-1* was obtained by using a divide and conquer approach. The resonances of fragments AF-1*265-340, Tau-5* (330 to 448) and AF-1* (142-448) were obtained by analyzing conventional three-dimensional triple resonance experiments acquired with standard Bruker pulse sequences on Bruker $600 \mathrm{MHz}$ and $800 \mathrm{MHz}$ spectrometers at $278 \mathrm{~K}$ in $20 \mathrm{mM}$ sodium phosphate buffer with $1 \mathrm{mM}$ TCEP at $\mathrm{pH}$ 7.4. The resonances of AF-1*265-340 and Tau- $5 *$ were equivalent to those of AF-1*, except for residues near the termini (Fig. S2), and allowed transferring the assignments from the former to the latter; the residues that were unique to $\mathrm{AF}-1 *$ were assigned directly by analysis of the relevant spectra. To measure the perturbations caused by EPI-001, EPI-002, EPI-003, EPI-004 and EPI-005 (details available as SI) on the resonances of AF-1* appropriate volumes of a 50 $\mathrm{mM}$ stock solution of these compounds in $100 \%$ dioxane- $\mathrm{d}_{8}$ were added to aliquots containing $25 \mu \mathrm{M} \mathrm{AF-1*}, 20 \mathrm{mM}$ sodium phosphate, $1 \mathrm{mM}$ TCEP, $10 \% \mathrm{D}_{2} \mathrm{O}, 30 \mu \mathrm{M}$ DSS$\mathrm{d}_{6}$ at $\mathrm{pH}$ 7.4. 


\section{Results and Discussion}

As previously reported5,6,15 the sequence of the NTD has features typically encountered in ID regions16. It has a high content of Gly, Pro, polar and charged residues and, as shown in figure $1 \mathrm{~b}$, possesses regions of low sequence complexity such as polyGln (residues 58-78, 84-89, 193-197), polyPro (372-379), polyAla (398-402) and polyGly (449-472) tracts. Such disordered tracts are commonly flanked by motifs of relatively low disorder propensity, with some helical propensity and rich in hydrophobic side chains, that are often involved in interactions with binding partners17. An analysis of the sequence of the NTD with predictors of disorder and helical propensity indicates that several such motifs are indeed present in the domain and it has been proposed that they may correspond to the regions of sequence recognized by general transcription factors and transcriptional co-regulators 15 (Fig. 1c,d,e).

A number of studies have aimed at identifying the regions of sequence of AF-1 that are essential for transcriptional activity 18,19 . Two large regions of sequence, known as transcription activation units 1 and 5 (Tau-1, 102-371 and Tau-5, 361-537) have emerged as especially important (Fig. 1b). Tau-1 is crucial for the transcriptional activity of AR when the receptor is activated by androgens 18 . Although Tau- 5 is less well characterised, it has been shown to be more important than Tau-1 when activation occurs via androgen independent mechanisms in androgen depletion independent cell lines derived from CRPC patients and mouse xenograft models of PC20,21. Efforts have also been directed at identifying the critical stretches of residues within these regions and suggest that for Tau-1 they correspond to residues 174 to 204 (core Tau-1)19, and for Tau-5 to residues 433 to 437 (WHTLF motif)20 (Fig. 1c).

\section{AF-1* is partially folded}

To investigate the structural properties of the NTD and its interaction with EPI-001 we cloned, expressed, purified and studied by NMR at 278K a 308-residue construct (AF-1*, residues 142-448) containing the part of AF-1 predicted to have a low disorder propensity (Fig. $1 \mathrm{~b}$ and 1c), that is flanked by polyGln and polyGly tracts (Fig. 1b). In agreement with previous reports based on the use of other biophysical methods 6 the ${ }^{1} \mathrm{H},{ }^{15} \mathrm{~N}-\mathrm{HSQC}$ spectrum of AF-1* had the features expected in an ID region such as low $\mathrm{H}^{\mathrm{N}}$ chemical shift dispersion (Fig. 2a). In addition, and contrary to what is the case for NTD constructs containing residues 1 to 141, which include the polyQ tract (Fig. 1b), this region of the NTD was sufficiently stable to allow a characterization of its structural properties by solution NMR.

In spite of its ID nature, the AF-1 region of the NTD has been shown to have helical propensity by circular dichroism (CD) in buffer and in the presence of cosolvents that stabilize intramolecular hydrogen bonds, as well as in the presence of the natural osmolyte trimethylamine oxide (TMAO)5,22. To identify the regions of sequence that adopt this secondary structure we assigned the resonances of AF-1* by a divide and conquer approach (see Methods), compared the $\mathrm{Ca}$ and $\mathrm{C} \beta$ chemical shifts, which are reliable reporters of secondary structure, to those predicted for disordered AF-1* (Fig. 2b) 23 and used the SSP algorithm of Forman-Kay and co-workers 24 to quantify the secondary structure propensity 
for the various residues of this construct from analysis of the backbone $\left({ }^{13} \mathrm{Ca},{ }^{13} \mathrm{C} \beta,{ }^{13} \mathrm{CO},{ }^{15} \mathrm{~N}, \mathrm{H}^{\mathrm{N}}\right)$ chemical shifts.

The results that we obtained indicated the presence of two regions of high helical propensity (defined as $\Delta \delta \mathrm{Ca}-\Delta \delta \mathrm{C} \beta>1 \mathrm{ppm}$ and SSP $\approx 0.5$, corresponding to a helical propensity of $50 \%$ ) which correspond to residues 185-200, in Tau-1, and 390-410, in Tau-5. Other regions of intermediate helical propensity (defined as $\Delta \delta \mathrm{Ca}-\Delta \delta \mathrm{C} \beta \approx 0.5 \mathrm{ppm}$ and SSP $\approx 0.2$ ) could be also identified, such as the region 230-240, in Tau-1, and 355-365 in Tau-5 (Fig. 2b, c). In addition to identifying regions of helical secondary structure the analysis of the chemical shifts also suggests that residues 144-154 and 270-290 of AF-1* adopt an extended conformation $(\Delta \delta \mathrm{Ca}-\Delta \delta \mathrm{C} \beta \approx-0.5 \mathrm{ppm}$ and $\mathrm{SSP} \approx-0.3)$ (Fig. $2 \mathrm{~b}, \mathrm{c}$ ).

To further characterize the structural properties of AF- $1 *$ we characterized the dynamics of AF-1* by measuring the transverse relaxation rates $\left(\mathrm{R}_{2}\right)$ of the backbone ${ }^{15} \mathrm{~N}$ nuclei. Such relaxation rates are good reporters of nascent secondary structure and transient tertiary contacts in chemically denatured and ID proteins 25 . The results that we obtained, presented in figure $2 \mathrm{~d}$, indicate that the regions with nascent secondary structure revealed by the analysis of the ${ }^{13} \mathrm{C}$ chemical shifts (Fig. $2 \mathrm{~b}$ ) also display relatively high $\mathrm{R}_{2}$ values. These are especially high, reaching values of $\mathrm{ca} 15 \mathrm{~s}^{-1}$, for three regions of sequence found in Tau-5 predicted to have low disorder propensity 26 and presenting, in two cases, high helical propensity.

Some of the regions of sequence that we identified as partially folded in AF- $1 *$ correspond in fact to the epitopes of binding partners of AR or are known to be important for the function of this receptor. In Tau-1, for example, the region that is most structured corresponds to core Tau-1 (Fig. 2b). This region encompases the motif ${ }^{183}$ LSEASTMQLL ${ }^{192}$, which is the binding epitope of TAB2, a component of the NCoR corepressor complex27. Partially folded residues 144 to 154, also in Tau-1, are part of the binding epitope of the amino-terminal bromodomains of BRD4 28. The region containing residues 230 to 240 , of intermediate helicity, overlaps with the binding site of CHIP, a protein known to recruit the chaperone machinery by interaction with Hsc70, Hsp70 and Hsp90, which mediates the degradation of AR by the proteasome 29 (Fig. 1e).

In Tau-5 the regions that we found to be partially folded are separated by motifs of low sequence complexity such as the Pro-rich region ${ }^{371}$ GPPPPPPPHP ${ }^{381}$ and the Gly-rich region ${ }^{414}$ GAGAAGPGSGSPS ${ }^{426}$. The former has been proposed to be the binding site of the SH3 domain of Src 30 and the latter harbors a phosphosite, S424, which is thought to be important for transcriptional activity 31 . Although we have not assigned the backbone resonances corresponding to the Pro residues in this Pro-rich motif, an analysis of the secondary chemical shifts of residues in its flanking regions (367-370, 385-388, Fig. 2b) suggests that it may adopt an extended conformation, likely of the polyproline II type, given the high Pro content; the polyproline II conformation is rather unusual but has been documented for several other IDPs. 32 The chemical shifts and transverse ${ }^{15} \mathrm{~N}$ relaxation rates of the Gly-rich region (414-426, Fig. 2b,c,d ) indicate, by contrast, that it is disordered, as expected due to its high Gly content. 
No binding partners have yet been identified for regions 355-365 and 390-410, that are partially helical, but the ${ }^{433}$ WHTLF $^{437}$ motif in region 433-447, corresponding to the core of Tau-5, has been proposed to bind both to activation function 2 (AF-2) in the LBD of androgen-bound AR 33,34 and to histone acetyltransferase p30035; in addition it has been shown to be indispensable for transcriptional activity in androgen depletion independent cell lines20.

We noted that the helical propensity of the partially folded regions identified in both Tau-1 and Tau-5 (SSP $\approx 0.5$, corresponding to a population of helix of ca 50\%) is substantially higher than that predicted by algorithms that solely consider local interactions such as Agadir 36 (Figs. 1e and 2b, c), indicating that non-local interactions may contribute to stabilizing the secondary structure. We thus investigated whether such non-local interactions included intramolecular contacts between residues in Tau-1 and Tau-5, similarly to what has been shown to occur in globular proteins under mild denaturing conditions37. For this analysis we used an NTD construct encompassing the part of Tau-5 contained in AF-1*, corresponding to residues 330-448 (termed Tau-5*, Fig. 1b). We compared the $\mathrm{H}^{\mathrm{N}}$ and ${ }^{15} \mathrm{~N}$ chemical shifts of Tau-5* to those of AF-1* and observed that they were highly similar (Fig. S2). These results indicate that the chemical environment that residues in the Tau-5 region experience is the same both in the presence and absence of Tau- 1 . We conclude that the secondary structure present in AF-1* does not rely on long-range interactions between residues in Tau- 1 and residues in Tau-5.

\section{EPI-001 interacts reversibly with Tau-5}

It has been proposed that the mechanism of action of EPI-001 (Fig. 3a) involves two steps 13. The first step being the formation of a reversible complex between this compound and a specific conformation of AF-1 and the second the nucleophilic attack, by a protein side chain, on the $\mathrm{C}-\mathrm{Cl}$ bond of EPI-001 to form an adduct unable to activate transcription. To investigate the first step of this mechanism and characterize the putative reversible complex we synthesized EPI-001 (details available as SI) and used NMR to analyze its effect on the resonances of AF-1* at $278 \mathrm{~K}$. As shown by MS under these conditions the irreversible reaction of EPI-001 with AF-1* is sufficiently slow to allow the study of the reversible interaction by NMR (Fig. S5).

The results that we obtained, shown in figures $3 \mathrm{~b}$ and $\mathrm{c}$, indicate that EPI-001 causes small but highly reproducible changes in ${ }^{15} \mathrm{~N}$ chemical shifts in residues 354 to 448 of AF-1*, that correspond to Tau-5. Interaction with EPI-001 affects the resonances of a large number of residues, which are found in the three regions of sequence in Tau-5 identified as partially folded by the combined analysis of the backbone ${ }^{13} \mathrm{C}$ chemical shifts and ${ }^{15} \mathrm{~N}$ transverse relaxation rates (Figs. 2b, 2d and 3b). We detected much smaller perturbations of the resonances of residues corresponding to Tau-1 even though this region contains partially helical regions such as residues in core Tau-1 (Figs. $2 \mathrm{~b}$ and $3 \mathrm{~b}$ ). These results indicate that EPI-001 does not simply interact with any region of sequence with helical propensity but, rather, that this compound targets Tau- 5 due to the ability of this sub-domain to adopt a conformation or, most likely, an ensemble of conformations that have affinity for it. 
With the aim of further investigating the nature of the interaction we monitored the effect of AF-1* on the resonances of EPI-001. We observed that sub-stoichiometric amounts ( $25 \mu \mathrm{M}$ and $50 \mu \mathrm{M}$ ) of AF-1* caused small but reproducible chemical shift changes in the ${ }^{1} \mathrm{H}$ NMR spectrum of EPI-001 at $250 \mu \mathrm{M}$, as well as increases in linewidth similar to those commonly observed in small drug-like molecules transiently associating with macromolecules (Fig. S3). Together with our observation of perturbations in a large number of residues of AF-1* this result suggests that EPI-001 interacts with an ensemble of conformations adopted by $\mathrm{AF}-1^{*}$ where these regions of sequence adopt a partially folded structure.

A large number of residues in Tau-5 experience chemical shift perturbations, more than what is expected for the binding of a small molecule. This can be due to the inspecific interaction of one or various molecules of EPI-001 with three independent interaction sites or to the combination of direct and indirect chemical shift changes due to structural changes occurring in Tau-5 upon interaction with EPI-00138. To exclude that one or more EPI-001 molecules interact independently with the three regions of sequence of AF-1* where chemical shift perturbations are observed we monitored the effect on its ${ }^{1} \mathrm{H}$ NMR spectrum of adding three peptides (R1 to R3) with sequences corresponding to regions 341-371, 391-414 and 426-446, respectively (details available as SI). We found that these peptides caused no changes in the spectrum of EPI-001, indicating that EPI-001 does not interact with them (Fig. S3) and confirming that residues 354-448 must simultaneously be present because they are necessary for binding this compound or for stabilizing the bound state of Tau-5* (Fig. 4).

It is interesting to note that Tau-5, the region of sequence of the NTD targeted by EPI-001, is partially folded, but it is important to emphasize that the data that we have obtained do not provide us with a mechanism of molecular recognition. It is possible that a conformational selection mechanism operates i.e. that EPI-001 interacts with a subset of the conformations that Tau-5* samples in equilibrium, but also that EPI-001 induces a new conformation in Tau-5*. Further work will be necessary to differentiate these two scenarios.

EPI-001 has two stereogenic centers and can therefore be found as four stereoisomers. To investigate whether the interaction between this compound and Tau-5 is stereospecific we synthesized the four stereoisomers (details available as SI) and studied their interaction with AF-1* by NMR. The results that we obtained (Fig. S4) indicate that the four compounds can interact with the NTD of AR, and thus the interaction appears to occur with little or no stereoselectivity. These results are in agreement with results obtained in vivo by Myung et al, who found that, although one of the stereoisomers tested was slightly more active than the other ones, the inhibitory activity of the four stereoisomers was similar.13

Put together our results indicate that AF-1 is partially folded in regions of sequence that correspond to those which are functionally relevant for interacting with the transcription machinery and co-regulators of transcription. In addition, they reveal that Tau- 1 and Tau-5, the two independent transcription activation units that are found in AF-1, correspond to different sub-domains that appear not to be involved in long-range interactions i.e. are structurally and dynamically, at least under our conditions, independent. Finally, and most importantly, they show that Tau-5, that plays a particularly important role in AR activation in 
the absence of androgens, can be targeted by compounds such as EPI-001 (Fig. 4). Although the lack of stereoselectivity that we observe suggests that the binding mode of EPI-001 may not be sufficiently well-defined for conventional drug development our results are of relevance for drug discovery for CRPC because they suggests that the NTD, and Tau- 5 in particular, may represent a suitable therapeutic target.

\section{Supplementary Material}

Refer to Web version on PubMed Central for supplementary material.

\section{Acknowledgements}

We thank J. M. Valverde (IRB) as well as the NMR facilities of the University of Barcelona (CCiT UB) and the Instituto de Química Física Rocasolano (IQFR, CSIC) for their assistance in, respectively, protein production and NMR. This work was supported by IRB, ICREA (X.S.), Obra Social "la Caixa" (Fellowship to E.D.M. and CancerTec grants to X.S.) MICINN (CTQ2009-08850 to X.S.), MINECO (BIO2012-31043 to X.S.; CTQ2014-56361-P to A.R), Marató de TV3 (102030 to X.S. and 102031 to E.E.P) the COFUND programme of the European Commission (C.T.W.P., A. R. and X.S.), the European Research Council (CONCERT, contract number 648201, to X.S.), the Ramón y Cajal program of MICINN (RYC-2011-07873 to C.W.B.) the Serra Hunter Programme (E.E.P.) and AGAUR (SGR-2014-56RR14 to E.E.P). IRB Barcelona is the recipient of a Severo Ochoa Award of Excellence from MINECO (Government of Spain).

\section{References}

(1). Gelmann EP. Molecular Biology of the Androgen Receptor. J Clin Oncol. 2002; 20:3001-3015. [PubMed: 12089231]

(2). Akaza H, Hinotsu S, Usami M, Arai Y, Kanetake H, Naito S, Hirao Y. Combined androgen blockade with bicalutamide for advanced prostate cancer. Cancer. 2009; 115:3437-3445. [PubMed: 19536889]

(3). Harris WP, Mostaghel EA, Nelson PS, Montgomery B. Androgen deprivation therapy: progress in understanding mechanisms of resistance and optimizing androgen depletion. Nat Clin Pract Urol. 2009; 6:76-85. [PubMed: 19198621]

(4). Karantanos T, Corn PG, Thompson TC. Prostate cancer progression after androgen deprivation therapy: mechanisms of castrate resistance and novel therapeutic approaches. Oncogene. 2013; 32:5501-5511. [PubMed: 23752182]

(5). Reid J, Kelly SM, Watt K, Price NC, McEwan IJ. Conformational analysis of the androgen receptor amino-terminal domain involved in transactivation. Influence of structure-stabilizing solutes and protein-protein interactions. J Biol Chem. 2002; 277:20079-20086. [PubMed: 11896058]

(6). Lavery DN, McEwan IJ. Structural characterization of the native NH2-terminal transactivation domain of the human androgen receptor: a collapsed disordered conformation underlies structural plasticity and protein-induced folding. Biochemistry. 2008; 47:3360-3369. [PubMed: 18284208]

(7). Quayle SN, Mawji NR, Wang J, Sadar MD. Androgen receptor decoy molecules block the growth of prostate cancer. Proc Natl Acad Sci U S A. 2007; 104:1331-1336. [PubMed: 17227854]

(8). Lamberto GR, Torres-Monserrat V, Bertoncini CW, Salvatella X, Zweckstetter M, Griesinger C, Fernandez CO. Toward the discovery of effective polycyclic inhibitors of alpha-synuclein amyloid assembly. J Biol Chem. 2011; 286:32036-32044. [PubMed: 21795682]

(9). Krishnan N, Koveal D, Miller DH, Xue B, Akshinthala SD, Kragelj J, Jensen MR, Gauss C-M, Page R, Blackledge M, Muthuswamy SK, et al. Targeting the disordered C terminus of PTP1B with an allosteric inhibitor. Nat Chem Biol. 2014; 10:558-566. [PubMed: 24845231]

(10). Metallo SJ. Intrinsically disordered proteins are potential drug targets. Curr Opin Chem Biol. 2010; 14:481-488. [PubMed: 20598937]

(11). Heller GT, Sormanni P, Vendruscolo M. Targeting disordered proteins with small molecules using entropy. Trends Biochem Sci. 2015; 40:491-496. [PubMed: 26275458] 
(12). Andersen RJ, Mawji NR, Wang J, Wang G, Haile S, Myung J-K, Watt K, Tam T, Yang YC, Bañuelos CA, Williams DE, et al. Regression of castrate-recurrent prostate cancer by a smallmolecule inhibitor of the amino-terminus domain of the androgen receptor. Cancer Cell. 2010; 17:535-546. [PubMed: 20541699]

(13). Myung J-K, Bañuelos CA, Fernandez JG, Mawji NR, Wang J, Tien AH, Yang YC, Tavakoli I, Haile S, Watt K, McEwan IJ, et al. An androgen receptor N-terminal domain antagonist for treating prostate cancer. J Clin Invest. 2013; 123:2948-2960. [PubMed: 23722902]

(14). Neidhardt FC, Bloch PL, Smith DF. Culture medium for enterobacteria. J Bacteriol. 1974; 119:736-747. [PubMed: 4604283]

(15). McEwan IJ. Intrinsic disorder in the androgen receptor: identification, characterisation and drugability. Mol Biosyst. 2012; 8:82-90. [PubMed: 21822504]

(16). Dunker AK, Lawson JD, Brown CJ, Williams RM, Romero P, Oh JS, Oldfield CJ, Campen AM, Ratliff CM, Hipps KW, Ausio J, et al. Intrinsically disordered protein. J Mol Graph Model. 2001; 19:26-59. [PubMed: 11381529]

(17). Mohan A, Oldfield CJ, Radivojac P, Vacic V, Cortese MS, Dunker AK, Uversky VN. Analysis of molecular recognition features (MoRFs). J Mol Biol. 2006; 362:1043-1059. [PubMed: 16935303]

(18). Jenster G, van der Korput HA, Trapman J, Brinkmann AO. Identification of two transcription activation units in the N-terminal domain of the human androgen receptor. J Biol Chem. 1995; 270:7341-7346. [PubMed: 7706276]

(19). Callewaert L, Van Tilborgh N, Claessens F. Interplay between two hormone-independent activation domains in the androgen receptor. Cancer Res. 2006; 66:543-553. [PubMed: 16397271]

(20). Dehm SM, Regan KM, Schmidt LJ, Tindall DJ. Selective role of an NH2-terminal WxxLF motif for aberrant androgen receptor activation in androgen depletion independent prostate cancer cells. Cancer Res. 2007; 67:10067-10077. [PubMed: 17942941]

(21). Metzger E, Müller JM, Ferrari S, Buettner R, Schüle R. A novel inducible transactivation domain in the androgen receptor: implications for PRK in prostate cancer. EMBO J. 2003; 22:270-280. [PubMed: 12514133]

(22). Kumar R, Betney R, Li J, Thompson EB, McEwan IJ. Induced alpha-helix structure in AF1 of the androgen receptor upon binding transcription factor TFIIF. Biochemistry. 2004; 43:3008-3013. [PubMed: 15023052]

(23). Tamiola K, Acar B, Mulder FAA. Sequence-specific random coil chemical shifts of intrinsically disordered proteins. J Am Chem Soc. 2010; 132:18000-18003. [PubMed: 21128621]

(24). Marsh JA, Singh VK, Jia Z, Forman-Kay JD. Sensitivity of secondary structure propensities to sequence differences between alpha- and gamma-synuclein: implications for fibrillation. Protein Sci. 2006; 15:2795-2804. [PubMed: 17088319]

(25). Klein-Seetharaman J, Oikawa M, Grimshaw SB, Wirmer J, Duchardt E, Ueda T, Imoto T, Smith LJ, Dobson CM, Schwalbe H. Long-range interactions within a nonnative protein. Science. 2002; 295:1719-1722. [PubMed: 11872841]

(26). Romero P, Obradovic Z, Li X, Garner EC, Brown CJ, Dunker AK. Sequence complexity of disordered protein. Proteins: Struct Funct Bioinf. 2001; 42:38-48.

(27). Zhu P, Baek SH, Bourk EM, Ohgi KA, Garcia-Bassets I, Sanjo H, Akira S, Kotol PF, Glass CK, Rosenfeld MG, Rose DW. Macrophage/cancer cell interactions mediate hormone resistance by a nuclear receptor derepression pathway. Cell. 2006; 124:615-629. [PubMed: 16469706]

(28). Asangani IA, Dommeti VL, Wang X, Malik R, Cieslik M, Yang R, Escara-Wilke J, WilderRomans K, Dhanireddy S, Engelke C, Iyer MK, et al. Therapeutic targeting of BET bromodomain proteins in castration-resistant prostate cancer. Nature. 2014; 510:278-282. [PubMed: 24759320]

(29). He B, Bai S, Hnat AT, Kalman RI, Minges JT, Patterson C, Wilson EM. An androgen receptor NH2-terminal conserved motif interacts with the COOH terminus of the Hsp70-interacting protein (CHIP). J Biol Chem. 2004; 279:30643-30653. [PubMed: 15107424] 
(30). Migliaccio A, Castoria G, Giovannelli P, Auricchio F. Cross talk between epidermal growth factor (EGF) receptor and extra nuclear steroid receptors in cell lines. Mol Cell Endocrinol. 2010; 327:19-24. [PubMed: 20603181]

(31). Gioeli D, Ficarro SB, Kwiek JJ, Aaronson D, Hancock M, Catling AD, White FM, Christian RE, Settlage RE, Shabanowitz J, Hunt DF, et al. Androgen receptor phosphorylation. Regulation and identification of the phosphorylation sites. J Biol Chem. 2002; 277:29304-29314. [PubMed: 12015328]

(32). Adzhubei AA, Sternberg MJE, Makarov AA. Polyproline-II helix in proteins: structure and function. J Mol Biol. 2013; 425:2100-2132. [PubMed: 23507311]

(33). He B, Kemppainen JA, Wilson EM. FXXLF and WXXLF sequences mediate the NH2-terminal interaction with the ligand binding domain of the androgen receptor. J Biol Chem. 2000; 275:22986-22994. [PubMed: 10816582]

(34). Hur E, Pfaff SJ, Payne ES, Grøn H, Buehrer BM, Fletterick RJ. Recognition and accommodation at the androgen receptor coactivator binding interface. PLoS Biol. 2004; 2:E274. [PubMed: 15328534]

(35). Lagarde WH, Blackwelder AJ, Minges JT, Hnat AT, French FS, Wilson EM. Androgen receptor exon 1 mutation causes androgen insensitivity by creating phosphorylation site and inhibiting melanoma antigen-A11 activation of NH2- and carboxyl-terminal interaction-dependent transactivation. J Biol Chem. 2012; 287:10905-10915. [PubMed: 22334658]

(36). Muñoz V, Serrano L. Elucidating the folding problem of helical peptides using empirical parameters. Nat Struct Biol. 1994; 1:399-409. [PubMed: 7664054]

(37). Bruun SW, Iešmantavičius V, Danielsson J, Poulsen FM. Cooperative formation of native-like tertiary contacts in the ensemble of unfolded states of a four-helix protein. Proc Natl Acad Sci U S A. 2010; 107:13306-13311. [PubMed: 20624986]

(38). Fenwick RB, Esteban-Martín S, Salvatella X. Understanding biomolecular motion, recognition, and allostery by use of conformational ensembles. Eur Biophys J. 2011; 40:1339-1355. [PubMed: 22089251]

(39). Shaffer PL, Jivan A, Dollins DE, Claessens F, Gewirth DT. Structural basis of androgen receptor binding to selective androgen response elements. Proc Natl Acad Sci U S A. 2004; 101:47584763. [PubMed: 15037741] 
a)

a)

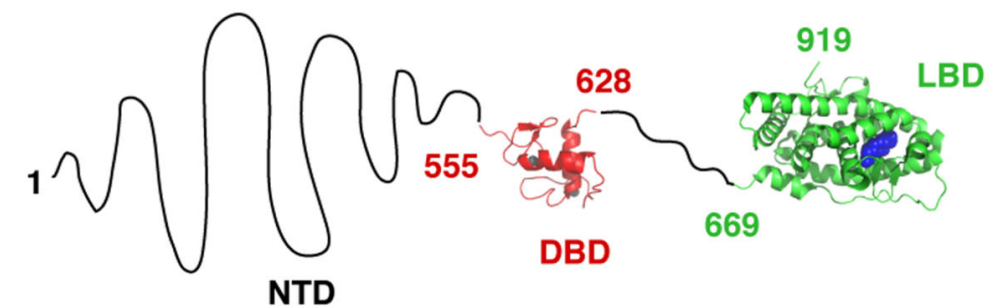

b)

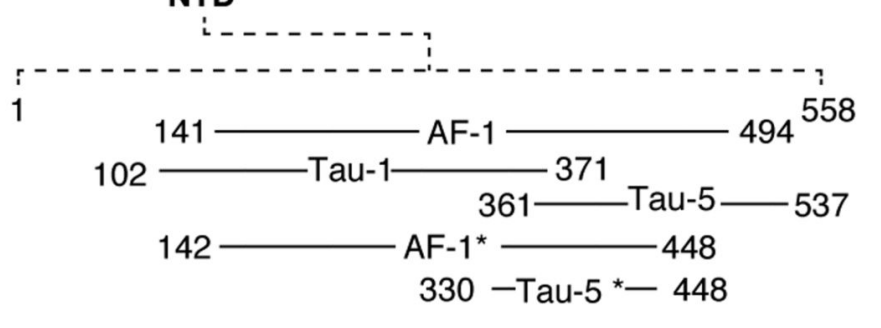

c)

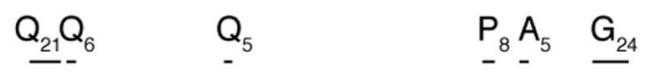

)

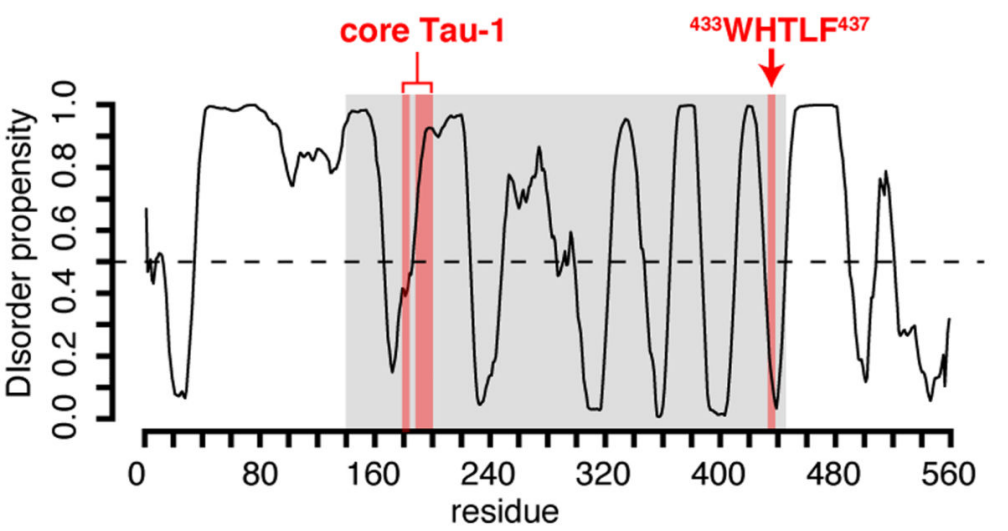

d)

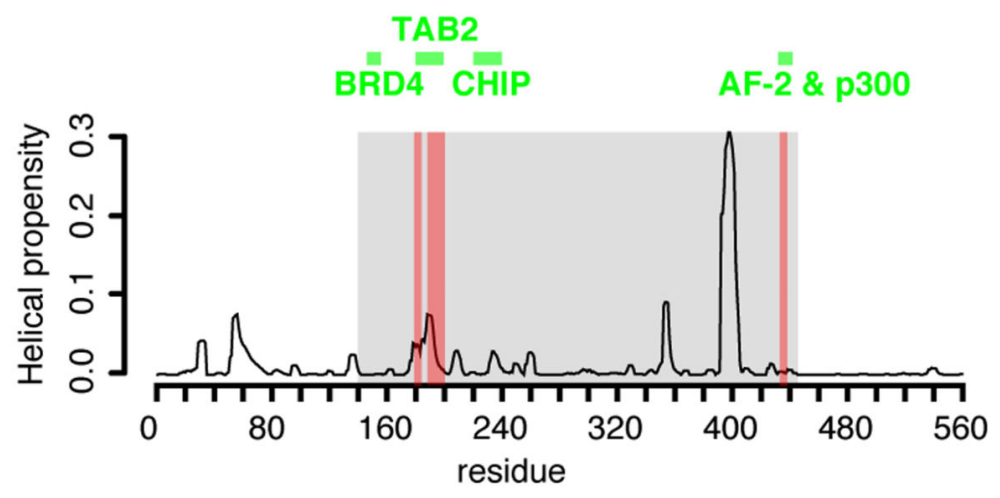

e)

Figure 1.

Predicted properties of the sequence of the transactivation domain of AR a) Domain organization of AR 34,39 with an indication of the position of $\mathrm{Zn}$ atoms in the DBD (grey) and of dihydrotestosterone (DHT) in the LBD (blue). b) Definitions of activation function 1 (AF-1), transcription acctivation units 1 and 5 (Tau-1 and Tau-5), AF- $1^{*}$ and Tau- $5 *$ (the regions of sequence studied in this work) with an indication of the regions of low sequence complexity such as polyGln $\left(\mathrm{Q}_{\mathrm{n}}\right)$, polyPro $\left(\mathrm{P}_{\mathrm{n}}\right)$, polyAla $\left(\mathrm{A}_{\mathrm{n}}\right)$ and polyGly $\left(\mathrm{G}_{\mathrm{n}}\right)$ tracts. c) Propensity to disorder of the NTD predicted by PONDR VL-XT26 with an indication of the 
functional motifs defining the core of Tau- 1 and Tau-5, shaded in red and of the region of sequence studied in this work, shaded in grey. d) Positions of the motifs of the NTD of AR involved in protein protein interactions and acronyms of the binding partners (see main text for details). e) Propensity to adopt a-helical conformations predicted by Agadir 36, as a function of residue number, with an indication of the core of Tau- 1 and Tau-5 (shaded in red) and AF-1* (shaded in grey). 

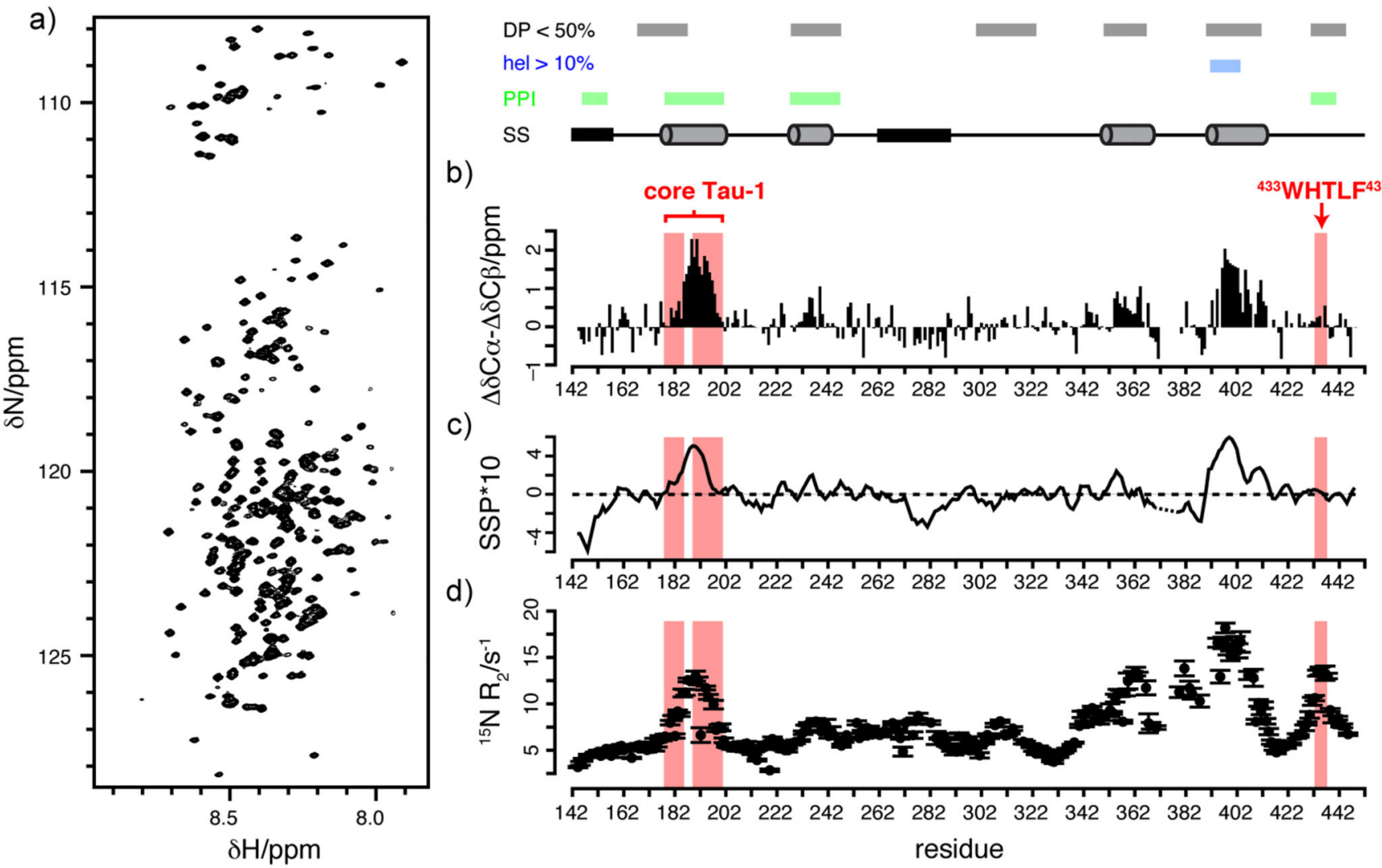

Figure 2.

Structural properties of the transactivation domain revealed by solution NMR a) ${ }^{1} \mathrm{H},{ }^{15} \mathrm{~N}$ HSQC spectrum of AF-1*. b) Plot of the difference between the secondary $\mathrm{Ca}$ and $\mathrm{C} \beta$ chemical shifts measured for AF- $1^{*}$ with an indication of the regions of sequence with predicted disorder propensity (DP) lower than 50\%, in grey, with predicted helical propensity (hel) higher than 10\%, in blue, and involved in protein protein interactions (PPIs), in green and, at the bottom of the top panel, an indication of the nascent secondary structure identified experimentally (SS, cylinders indicate helical propensity, black rectangles indicate propensity to adopt an extended conformation). c) Plot of the secondary structure propensity of the residues of $\mathrm{AF}-1^{*}$, where $\mathrm{SSP}=1$ indicates a fully formed helix and $\mathrm{SSP}=1$ an extended conformation, obtained by using the algorithm SSP to extract the information on secondary structure contained in backbone $\left({ }^{13} \mathrm{Ca},{ }^{13} \mathrm{C} \beta,{ }^{13} \mathrm{CO},{ }^{15} \mathrm{~N}, \mathrm{HN}\right)$ chemical shifts. d) Plot of the ${ }^{15} \mathrm{~N}$ transverse relaxation rates $\left(\mathrm{R}_{2}\right)$ of the residues of AF-1* at $250 \mu \mathrm{M}$. The cores of Tau- 1 and Tau-5 are shaded in red. 
a)<smiles>CC(C)(c1ccc(OCC(O)CO)cc1)c1ccc(OCC(O)CCl)cc1</smiles>

b)

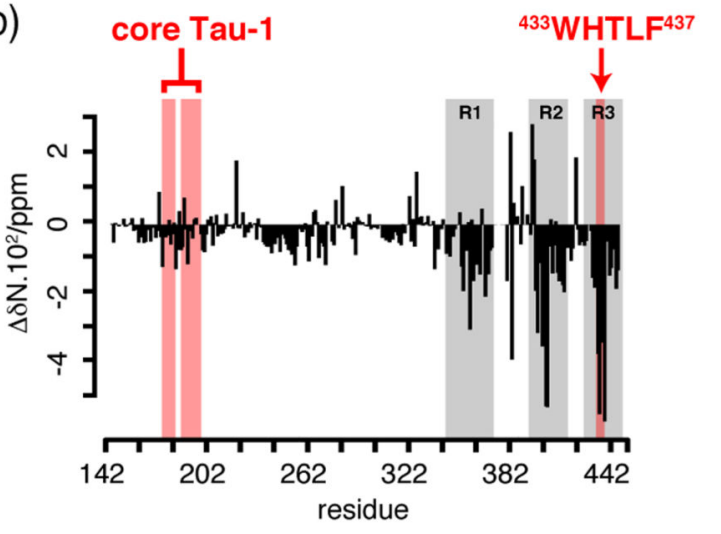

c)

Tau-1
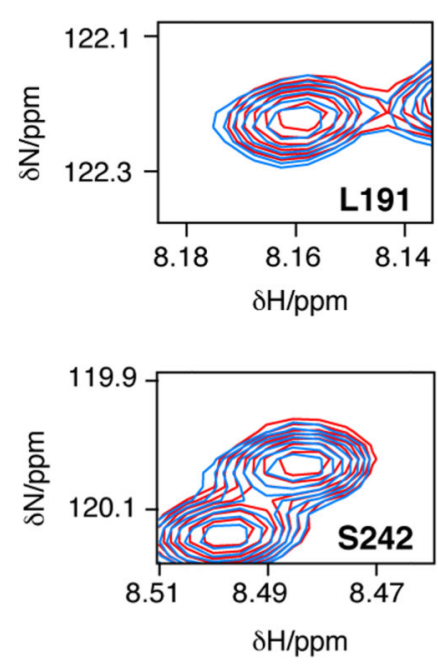

25 uM AF-1 * $+250 \mu \mathrm{M}$ EPI-001
Tau-5
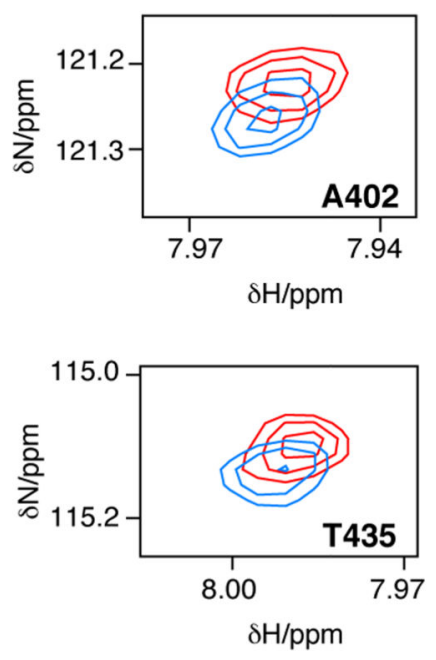

25 uM AF-1* $+250 \mu \mathrm{M}$ EPI-001

Figure 3.

EPI-001 selectively interacts with transcription activation unit 5 of the transactivation domain of AR a) Structure of EPI-001 with an indication of the two stereogenic centres with the symbol *. b) Plot, as a function of residue number, of the change in ${ }^{15} \mathrm{~N}$ chemical shift of AF- $1 *$ caused by addition of EPI-001. c) Selected regions of the ${ }^{1} \mathrm{H},{ }^{15} \mathrm{~N}-\mathrm{HSQC}$ spectrum of $25 \mu \mathrm{M} \mathrm{AF}-1 *$ in the absence (blue) and in the presence (red) of 10 molar equivalents of EPI-001. 


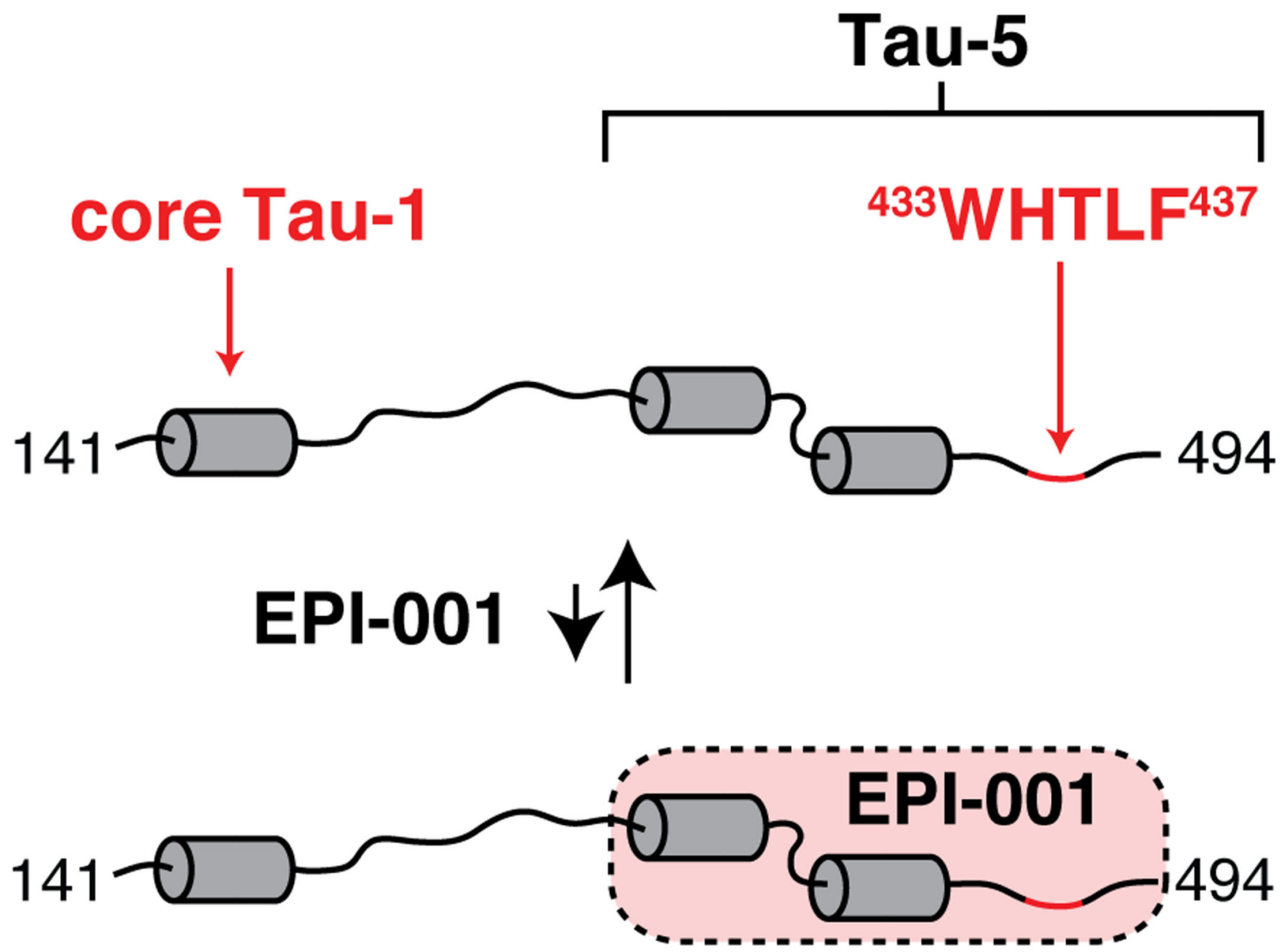

Figure 4.

Scheme of the interaction of EPI-001 with partially folded of Tau-5. 